\title{
Genomic characterization of pediatric B-lymphoblastic lymphoma and B-lymphoblastic leukemia using formalin-fixed tissues
}

\author{
Julia A. Meyer ${ }^{1,2}$ ～Delu Zhou ${ }^{3}$ ～Clinton C.Mason ${ }^{4}$ | Jonathan M.Downie ${ }^{5}$ | \\ Vladimir Rodic $^{3}$ | Minnie Abromowitch ${ }^{6}$ | Birte Wistinghausen ${ }^{7}$ | \\ Amanda M. Termuhlen $^{8}$ | Anne L. Angiolillo9 $\quad$ Sherrie L. Perkins ${ }^{3,10 ~ \mid ~}$ Mark A. Lones $^{11}$ \\ Phillip Barnette $^{4}$ | Joshua D. Schiffman ${ }^{1,2,4}$ | Rodney R. Miles ${ }^{2,3,10}$
}

${ }^{1}$ Department of Oncological Sciences, University of Utah, Salt Lake City, Utah

${ }^{2}$ Huntsman Cancer Institute, University of Utah, Salt Lake City, Utah

${ }^{3}$ Department of Pathology, University of Utah, Salt Lake City, Utah

${ }^{4}$ Department of Pediatrics, University of Utah, Salt Lake City, Utah

${ }^{5}$ Department of Human Genetics, University of Utah, Salt Lake City, Utah

${ }^{6}$ Department of Pediatrics, University of Nebraska Medical Center, Omaha, Nebraska

${ }^{7}$ Department of Pediatrics, Icahn School of Medicine at Mount Sinai, New York, New York

${ }^{8}$ Department of Pediatrics, Keck School of Medicine at the University of Southern California, Children's Hospital Los Angeles, Los Angeles, California

${ }^{9}$ Division of Oncology, Center for Cancer and Blood Disorders, Children's National Medical Center, Washington, District of Columbia

${ }^{10}$ ARUP Institute for Experimental Pathology, Salt Lake City, Utah

${ }^{11}$ Department of Pathology and Laboratory Medicine, University of California Los Angeles, Los Angeles, California

Correspondence

Rodney R. Miles, Department of Pathology, University of Utah and ARUP Laboratories, $15 \mathrm{~N}$. Medical Drive East, JMRB 2100, Salt Lake City, UT 84112-0565.

Email:rodney.miles@path.utah.edu

[This article was corrected on December 30, 2016, because coauthor Mark A. Lones needed to have his name included.]

\begin{abstract}
Background: Recurrent genomic changes in B-lymphoblastic leukemia (B-ALL) identified by genome-wide single-nucleotide polymorphism (SNP) microarray analysis provide important prognostic information, but gene copy number analysis of its rare lymphoma counterpart, Blymphoblastic lymphoma (B-LBL), is limited by the low incidence and lack of fresh tissue for genomic testing.

Procedure: We used molecular inversion probe (MIP) technology to analyze and compare copy number alterations (CNAs) in archival formalin-fixed paraffin-embedded pediatric B-LBL $(n=23)$ and B-ALL $(\mathrm{n}=55)$.

Results: Similar to B-ALL, CDKN2A/B deletions were the most common alteration identified in $6 / 23$ (26\%) B-LBL cases. Eleven of 23 (48\%) B-LBL patients were hyperdiploid, but none showed triple trisomies (chromosomes 4, 10, and 17) characteristic of B-ALL. IKZF1 and PAX5 deletions were observed in 13 and $17 \%$ of B-LBL, respectively, which was similar to the reported frequency in B-ALL. Immunoglobulin light chain lambda (IGL) locus deletions consistent with normal light chain rearrangement were observed in 5/23 (22\%) B-LBL cases, compared with only $1 \%$ in B-ALL samples. None of the B-LBL cases showed abnormal, isolated VPREB1 deletion adjacent to IGL locus, which we identified in $25 \%$ of B-ALL.
\end{abstract}

Conclusions: Our study demonstrates that the copy number profile of B-LBL is distinct from B-ALL, suggesting possible differences in pathogenesis between these closely related diseases.

\section{KEYWORDS}

genomics, leukemia, lymphoma, pediatrics
ABBREVIATIONS: B-ALL, B-lymphoblastic leukemia; B-LBL, B-lymphoblastic lymphoma; CNAs, copy number alterations; COG, Children's Oncology Group; FFPE, formalin-fixed paraffin-embedded; IGL, immunoglobulin light chain lambda; MIP, molecular inversion probe; SJCRH, St. Jude Children's Research Hospital; SNP, single-nucleotide polymorphism

\section{1 | BACKGROUND}

Most malignancies of B-lineage lymphoblasts present as Blymphoblastic leukemia (B-ALL), while only $10-20 \%$ present as B-lymphoblastic lymphoma (B-LBL). ${ }^{1}$ The distinction according to the 
2008 WHO classification is that B-ALL involves bone marrow with or without peripheral blood involvement, while B-LBL occurs in nodal or extranodal sites without significant bone marrow $(<25 \%$ blasts) or peripheral blood involvement. ${ }^{2}$ As B-ALL and B-LBL show similar morphology and immunophenotype, it has been suggested that these two represent different clinical manifestations of the same disease process. Pediatric B-LBL is currently treated according to B-ALL protocols with a prognosis comparable to that of low-risk B-ALL. ${ }^{3,4}$

Due to the accessibility of tumor cells, B-ALL is one of the best characterized neoplasms at the cytogenetic and molecular genetic levels resulting in important prognostic associations. The introduction of high-resolution single-nucleotide polymorphism (SNP) microarray technology has furthered the discovery of focal recurrent copy number alterations (CNAs) in B-ALL including CRLF2, IKZF1, JAK2, PAX5, and VPREB1 alterations ${ }^{5-7}$ and the association of certain gene deletions such as IKZF1 and VPREB1 with prognosis. ${ }^{8,9}$ However, genomic analysis of B-LBL is limited by the lack of fresh or frozen tissue specimens for cytogenetic and molecular analysis and genomic testing.

Formalin-fixed paraffin-embedded (FFPE) tissues are routinely stored in pathology archives and represent a source of tissue for studies of rare neoplasms or patient cohorts where fresh frozen samples were not collected. We previously demonstrated the feasibility of high-resolution CNA analysis in FFPE Burkitt lymphoma samples. ${ }^{10}$ FFPE bone marrow aspirate clot samples are routinely archived at many centers and could represent an abundant source of leukemia specimens, which have not previously been utilized in B-ALL SNP microarray studies. Although B-LBL FFPE tissue in blocks was scarce, we had access to FFPE tissue sections on unstained slides as a potential source of $B-L B L D N A$.

In this study, we utilized molecular inversion probe (MIP)-based SNP microarray technology to detect CNAs in clinically archived FFPE pediatric B-ALL and B-LBL samples. We compared CNAs identified in $B-L B L$ to those in B-ALL to determine if they possess similar genetic alterations. We also compared our FFPE B-ALL copy number results to those of fresh frozen B-ALL samples to determine if accurate CNA calls were generated. ${ }^{7}$ Our study demonstrates the utility of this assay for the study of archival tissue specimens, highlights the use of FFPE samples to identify focal genomic changes in leukemia and lymphoma, and importantly characterizes B-LBL recurrent CNAs, including changes that are distinct from B-ALL.

\section{2 | PROCEDURE}

\section{1 | Patients and samples}

The Institutional Review Board of the University of Utah approved this study. We obtained FFPE bone marrow aspirate clots from 55 B-ALL patients diagnosed at Primary Children's Hospital at the University of Utah from 2004 to 2009. B-LBL unstained slides submitted for central pathology review were available for patients enrolled in the Children's Oncology Group (COG) A5971 11 and AALL0932 trials and approved for use in this study by COG (Protocol \#ANHL15B1-Q) and the Cancer Therapy Evaluation Program CTEP. Patients enrolled in COG A5971 were treated as low risk if disease was localized and high risk for those
TAB LE 1 Clinical characteristics of each cohort

\begin{tabular}{|c|c|c|c|c|c|}
\hline \multicolumn{6}{|c|}{ Patient characteristics } \\
\hline & & $\begin{array}{c}\text { B-ALL } \\
(\mathrm{N}=55)\end{array}$ & $\%$ & $\begin{array}{c}\text { B-LBL } \\
(\mathrm{N}=23)\end{array}$ & $\%$ \\
\hline \multicolumn{6}{|c|}{ Gender } \\
\hline & Female & 28 & 51 & 15 & 65 \\
\hline & Male & 27 & 49 & 8 & 35 \\
\hline & Unknown & & & & \\
\hline \multicolumn{6}{|l|}{ Age } \\
\hline & Median (in years) & 6.5 & & 6.5 & \\
\hline & Less than 10 years & 38 & 69 & 15 & 65 \\
\hline & At least 10 years & 17 & 31 & 8 & 35 \\
\hline \multirow{3}{*}{$\begin{array}{l}\text { Risk } \\
\text { group }\end{array}$} & Low & 0 & & & \\
\hline & Standard & 33 & 60 & & \\
\hline & High & 22 & 40 & & \\
\hline
\end{tabular}

with disseminated disease. Unstained FFPE slides were stored at room temperature for 1-10 years. Our results were compared to a B-ALL cohort that included both standard and high-risk patients treated and analyzed by St. Jude Children's Research Hospital (SJCRH, N = 192). ${ }^{7}$ Leukemia and germline CEL files from the Affymetrix Genome-Wide Human SNP Array 6.0 and the Affymetrix GeneChip Human Mapping $500 \mathrm{~K}$ Array were obtained from GSE5511, and only B-ALL samples were utilized for comparison. See Table 1 for clinical characteristics of each cohort.

\section{2 | DNA extraction}

H\&E staining was reviewed on each case to evaluate tissue adequacy in clot sections and to verify at least $80 \%$ tumor cells in B-LBL sections. B-ALL bone marrow aspirate clots were required to contain at least $66 \%$ lymphoblasts based on the corresponding aspirate smear differential. FFPE tissue from 4 to 10 slides per B-LBL case $(n=30)$ was scraped into tubes, and 50- $\mu \mathrm{m}$ thick sections of B-ALL FFPE bone marrow aspirate clots $(n=55)$ were collected from archived tissue blocks. DNA was isolated using the RecoverAll Total Nucleic Acid Isolation Kit (Ambion/Applied Biosystems, Austin, Texas) and quantified with PicoGreen (Invitrogen, Waltham, Massachusetts). Total genomic DNA yields from the FFPE samples ranged from 19.8 up to $1,924.1 \mathrm{ng}$ (median $376.5 \mathrm{ng}$ ) for B-ALL. Of the initial 30 B-LBL cases, 23 yielded adequate DNA for MIP analysis; the six cases with insufficient DNA yields were bone or small skin biopsies. For B-LBL cases with adequate DNA, the yield ranged from 6.9 to $1,089.0 \mathrm{ng}$ (median $165.8 \mathrm{ng}$ ).

\subsection{Molecular inversion probe and copy number data analysis}

The OncoScan ${ }^{\text {TM }}$ FFPE Express assay (Affymetrix Inc., Santa Clara, California), with increased coverage for known cancer genes, was used on the samples from these Utah cohorts with hybridization and scanning as previously described. ${ }^{10}$ Data visualization, CNA calling, and analysis for the OncoScan ${ }^{\mathrm{TM}}$ FFPE Express Array as well as the Affymetrix Genome-Wide Human SNP 6.0 and $500 \mathrm{~K}$ array data 

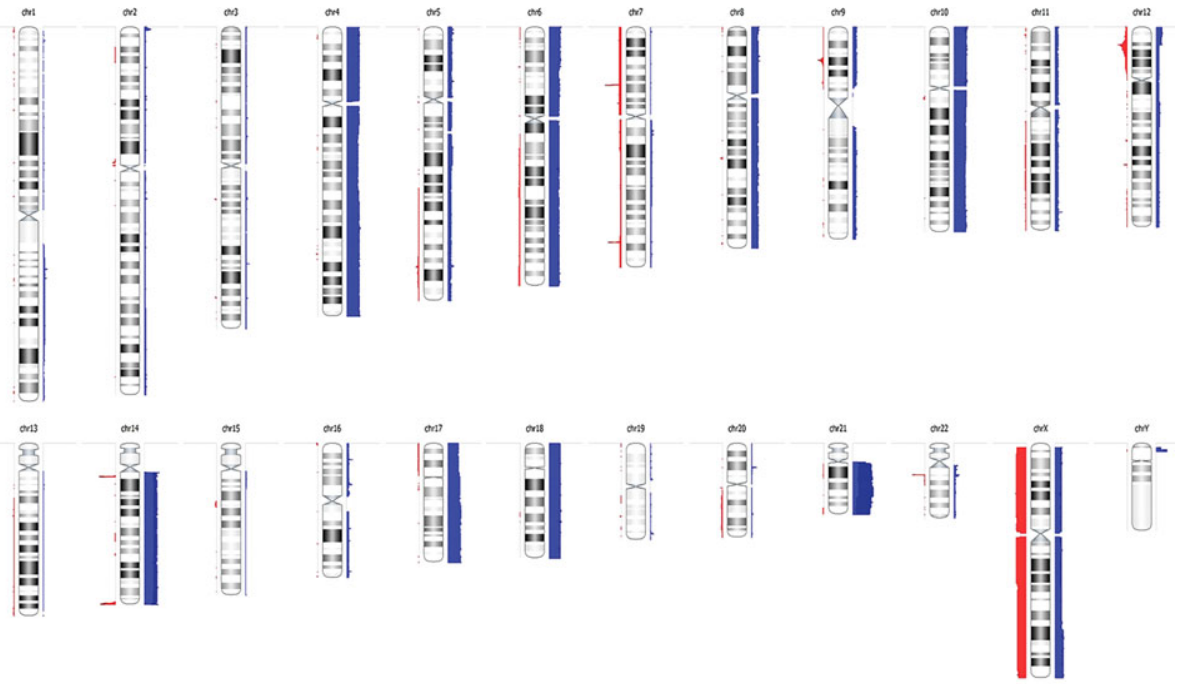

FIGURE 1 Whole genome view of B-ALL copy number abnormalities. Summary of SNP array copy number data from all 55 cases. Chromosomal gains are shown to right of the affected chromosome in blue and losses are to the left in red. Thicker bars indicate areas of recurrent change

were performed with Nexus Copy Number 7.5 (BioDiscovery, Inc., El Segundo, California). The copy number values of each microarray probe were loaded into Nexus with default settings without probe recentering. The median probe values of diploid regions of chromosomes 1-8 from each sample were used to re-center all probes. CNA genomic segments were identified using the default settings and calling parameters of BioDiscovery's SNP-FASST2 Segmentation Algorithm (a Hidden Markov Model based approach). Gene regions identified as loss or gain were then manually inspected to verify each call was supported by five or more probes and were $50 \mathrm{~kb}$ or more in length. This was necessary to allow greater consistency in copy number calls across chips with varying number of probes spanning certain genes. Peak prevalence of common events over identical regions was then compared in each data set. DNA gains and losses resulting from normal antigen receptor gene rearrangements at Chr2p11 (IGKL), Chr7p14 (TRGV), Chr7q34 (TRBV), Chr14q11-12 (TRAV, TRDV, TRDJ, TRDC, and TRAJ), Chr14q32 (IGHV) and Chr22q11.22 (IGLL) were not counted with the exception of focal deletions within the IGLL/VPREB1 region as previously described in B-ALL. ${ }^{9}$ Gene annotation was based on Build 36.1 of NCBI's human genome reference sequence for all B-ALL samples and Build 37 for all B-LBL samples. Copy number at TCF3 was also not assessed due to probe coverage differences across chips at this locus, which could not be rectified.

\section{4 | Statistics}

B-ALL vs. B-LBL genetic differences were compared using a two-tailed, Fisher exact test or two-tailed chi-square test where appropriate.

\section{3 | RESULTS}

\section{1 | Patient characteristics}

The median age of the B-LBL patients was 6 years (range 1-17) and 6.5 years for B-ALL patients (range 1-26) (Table 1). ALL cases were
TAB LE 2 ALL and LBL deletions across all cohorts (B-cell development genes, etc.)

\begin{tabular}{|lccc|} 
& $\begin{array}{c}\text { Utah } \\
\text { B-LBL (\%) }\end{array}$ & $\begin{array}{c}\text { Utah } \\
\text { B-ALL (\%) }\end{array}$ & $\begin{array}{c}\text { St. Jude } \\
\text { B-ALL (\%) }\end{array}$ \\
\hline Gene & $\mathbf{N}=\mathbf{2 3}$ & $\mathrm{N}=55$ & $\mathbf{N}=192$ \\
\hline CDKN2A/B & 26 & 20 & 33.9 \\
\hline EBF1 & 0 & 10.9 & 4.2 \\
\hline ETV6 & 13 & 29 & 26.6 \\
\hline IKZF1 & 13 & 12.7 & 8.9 \\
\hline PAX5 & 17 & 10.9 & 29.2 \\
\hline VPREB1 (region) & 4.3 & 25.4 & 28.6 \\
\hline $\begin{array}{l}\text { At least one } \\
\text { B-cell } \\
\text { development } \\
\text { gene }\end{array}$ & 26 & 43.6 & 53.6 \\
\hline
\end{tabular}

aB-cell development gene deletion examined: IKZF1, EBF1, PAX5, LEF1, $B L N K$, and VPREB1.

predominately NCI Standard Risk (60\% standard, 40\% high risk). B-LBL cases were all treated as low risk; stage at diagnosis and tumor site are provided in Supplementary Table S1.

\subsection{Recurrent CNVs in B-ALL}

The OncoScan ${ }^{\mathrm{TM}}$ FFPE Express $330 \mathrm{~K}$ cancer panel detected multiple CNAs of different sizes and frequencies in the FFPE B-ALL cohort of patients (Fig. 1 and Supplementary Table S2). The majority of the deletions in the FFPE B-ALL cohort have been previously reported in studies of fresh/frozen tissues and occurred at very similar rates across other B-ALL patients (Table 2). The most frequent deletions found in the B-ALL MIP analysis included commonly occurring deletions ETV6 (16/55, 29\%), VPREB1 (14/55, 25\%), CDKN2A/CDKN2B-AS1 (11/55, 20\%; eight homozygous, three hemizygous), IKZF1 (7/55, 13\%), PAX5 (6/55, 11\%), and EBF1 (6/55, 11\%). Deletions in other genes previously implicated in B-ALL were also observed at low frequency such as BTG1 $(6 / 55,11 \%)$ and $R B 1(2 / 55,4 \%) .7,8$ 


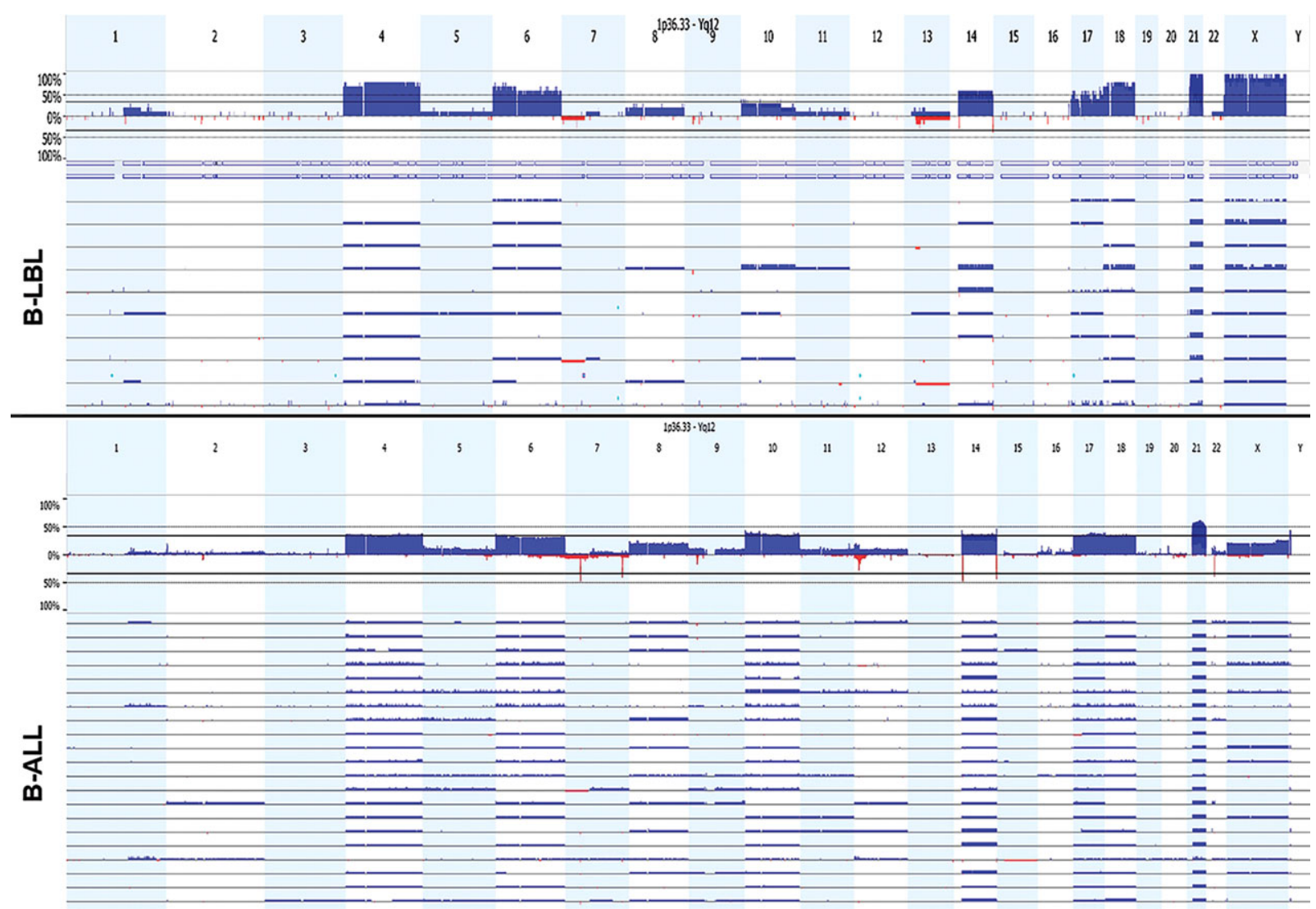

FIGURE 2 Chromosomal gains in hyperdiploid cases of B-LBL versus B-ALL. Each row represents a patient and is grouped according to disease. Chromosome numbers are listed across the top. Height of blue bars indicate the overall frequency of chromosomal gain in each patient population (red = deletion; blue $=$ gain)

Recurrent amplifications in B-ALL consisted largely of whole chromosomal amplifications. Our cohort contained previously reported trisomies present in hyperdiploid cases (Figs. 1 and 2). Overall, 21/55 (38\%) B-ALL cases were hyperdiploid and 17/21 cases had triple trisomies: $+4,+10$, and +17 (Supplementary Table S2).

\section{3 | Recurrent CNVs in B-LBL}

All 23 cases of B-LBL with adequate DNA for MIP analysis yielded interpretable copy number data. Four cases showed noise typical of specimens with low-quality DNA, but in each case, gain or loss of either large segments or entire chromosomes could still be determined reliably with matching B-allele frequencies for each CNV. Genome-wide copy number data were generated from all 23 cases (Fig. 3 and Supplementary Table S3), and 22 cases showed at least one gain or loss (96\%). The median number of gains and losses per case was 4 (range 0-9) and 1.5 (range $0-5$ ), respectively.

\section{4 | B-LBL show similarities and differences when compared to genomic copy number abnormalities present in Utah B-ALL cohort}

B-LBL showed many features commonly seen in B-ALL. CDKN2A/B deletions were identified in 6/23 (26\%) of the B-LBL and 11/55 (20\%) of our B-ALL cases. Similar rates of IKZF1 and PAX5 deletion were also seen. However, differences in ETV6 and EBF1 deletions were identified between the two groups with both genes having lower alteration frequencies in B-LBL (Table 2).

Hyperdiploidy is defined in WHO 2008 as more than 50 chromosomes, usually less than 66 , and with an incidence of about $25 \%$ in BALL. $^{2}$ Hyperdiploidy, involving chromosomes 4, 6, 10, 14, 17, 18, 21, and $X$ is found most often, with chromosomes 21 and $X$ the most frequently cited gains. ${ }^{12}$ Additionally, hyperdiploidy involving chromosomes 4,10 , and 17 is associated with a favorable prognosis. ${ }^{13} \mathrm{We}$ found hyperdiploidy involving trisomies of chromosomes 4, 6, 18, 21, and $X$ as the most frequent whole chromosome gains in B-LBL (Fig. 2 and Supplementary Table S2). Similar to B-ALL, gains of chromosome 21 and $X$ were the most frequent (10/10 B-LBL cases), while gains of chromosome 10 were seen the least $(3 / 10)$. None of the B-LBL cases harbored the characteristically favorable triple trisomy of 4,10 , and 17 and interestingly all cases of hyperdiploidy were found in patients with local versus disseminated disease $(P=0.0075)$.

\subsection{B-LBLs show a lower frequency of B-cell development gene deletions and different immunoglobulin light chain lambda locus deletions compared with B-ALL}

In B-ALL, gene copy number changes in the genes regulating B-cell development were found in about $43-54 \%$ of cases (Table 2). These genes included IKZF1, PAX5, EBF1 (transcription factors), BLNK, LEF1, 


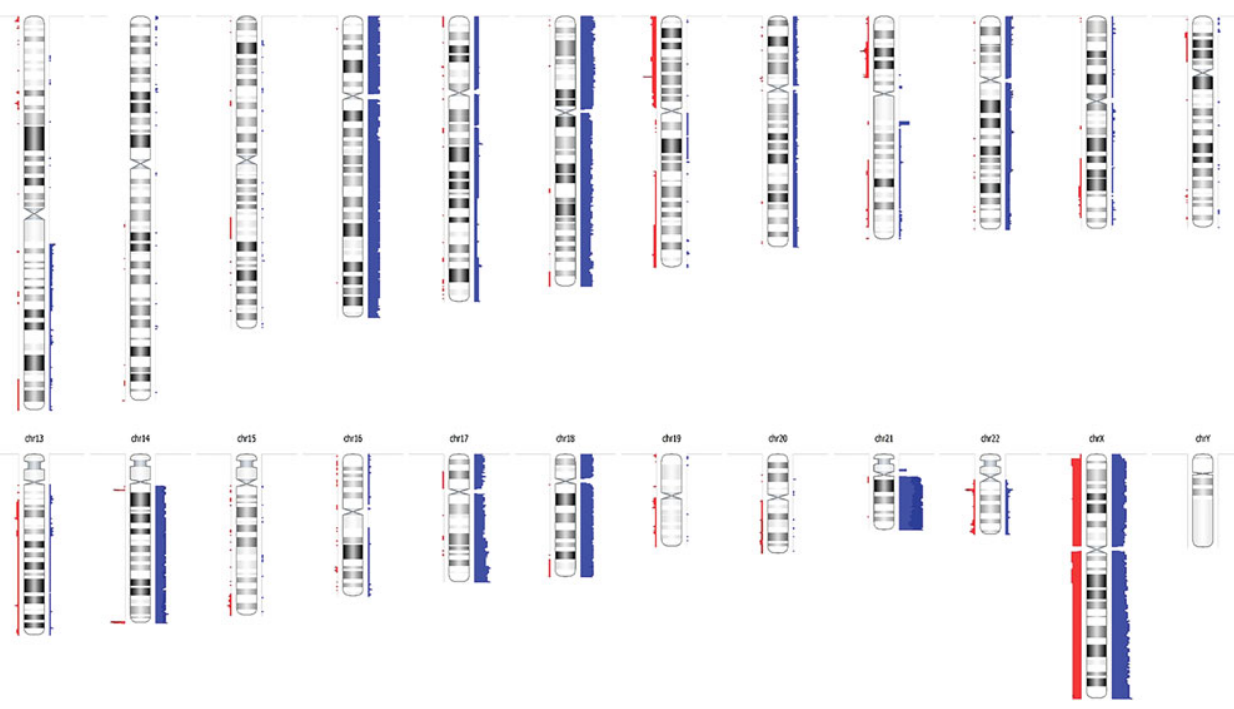

FIGURE 3 Whole genome view of B-LBL copy number abnormalities. Summary of SNP array copy number data from all 23 cases. Chromosomal gains are shown to right of the affected chromosome in blue and losses are to the left in red. Thicker bars indicate areas of recurrent change

and VPREB1 (encodes surrogate light chain). In B-LBL, gene deletions (IKZF1 and/or PAX5) were observed in similar percentages to B-ALL, while the deletions of EBF1 and VPREB1 were rarely seen (Table 2). When considered together, deletion of any one or more of the B-cell development genes occurred in $26 \%$ of $B-L B L$ versus $49 \%$ in B-ALL (average in combined Utah and SJCRH cohort, $P=0.024$ ). Additionally, no differences were seen between the frequencies of B-cell development gene deletions occurring in disseminated versus local B-LBL disease.

B-LBL also differed from B-ALL in the pattern of deletions involving the immunoglobulin light chain lambda (IGL) locus. As we have shown in our previous studies, SNP arrays in mature B-cell lymphomas demonstrate contiguous IGL locus deletions that extend to the VJ junction, consistent with normal light chain rearrangement, while BALLs show abnormal, focal IGL deletions that do not extend to the VJ junction and are thus not consistent with light chain rearrangement. ${ }^{9}$ B-LBL showed contiguous IGL deletions indicating light chain rearrangement in $5 / 23(22 \%)$, compared with only $1 / 55(2 \%)$ in the B-ALL FFPE cohort ( $P=0.0076$, two tailed Fisher exact; Fig. 4). Only one of the B-LBL cases showed a focal IGL deletion on or upstream of the VPREB1 gene, which we have recently identified was present in $25 \%$ of B-ALL (Table 2, $P=0.05$ ). ${ }^{9}$ While the deletion in this patient did not reach the $\mathrm{V}$-J junction characteristic of IGLL rearrangement, the deleted segment also did not match the boundaries often seen in B-ALL (either upstream of VPREB1 or directly centered on VPREB1).

\section{CONCLUSIONS}

High-resolution, genome-wide SNP array profiling of B-ALL has provided important prognostic and biologic insights. ${ }^{7,8}$ The biology of $\mathrm{B}$ $\mathrm{LBL}$ is much less characterized than that of B-ALL because it is much less common and fresh tissue is rarely available; there are only a few studies of B-LBL genomics. ${ }^{3,14,15}$ The ability to study rare diseases such as B-LBL is often further limited by availability of fresh frozen tissue required for many genomic techniques, so development of methods for analysis of fixed tissues from rare diseases is advantageous. Using a MIP-based SNP array, we demonstrated the feasibility of analyzing clinically archived FFPE bone marrow aspirate clot specimens and tissue from unstained glass slides using SNP microarrays. We identified previously reported CNAs in FFPE samples from a local B-ALL cohort and demonstrated that CNAs occurred at frequencies similar to another previously published cohort of fresh-frozen B-ALL samples. This allowed us to perform the largest high-resolution SNP array study of $B-L B L$ to date and directly compare the genomic features of $B-L B L$ to $B-A L L$ using the same platform. The FFPE samples in our study were up to 10 years old and still performed remarkably well on the MIP platform with high-resolution results for copy number data.

Many of the most frequent CNAs identified in our cohort were reported previously in studies using fresh or frozen B-ALL samples, thus validating the CNAs that we describe and the MIP assay itself. CNAs affect multiple cellular pathways in B-ALL and are commonly found within genes that regulate B-cell development and differentiation, such as EBF1, PAX5, IKZF1, and VPREB1. ${ }^{7-9}$ Also consistent with previous reports in $B-A L L$, we identified recurrent deletions involving ETV6, CDKN2A, and BTG1. ${ }^{16-18}$ Of note, to increase CNA calling consistency across different microarray versions, we only utilized CNA calls across genes if the region of aberration was at least $50 \mathrm{~kb}$. The presence of smaller micro-aberrations could potentially increase the prevalence rates we observed.

In contrast to B-ALL, genomic studies of B-LBL are few in number and include small numbers of cases. Maitra et al. ${ }^{3}$ reported cytogenetic results from eight B-LBL cases, which lacked the characteristic translocations of B-ALL. They found one case with hyperdiploidy (>50 chromosomes) and three other cases with additional chromosome 21 material. A more recent study of lymphoblastic lymphoma karyotypes included two patients with B-LBL. Both patients 


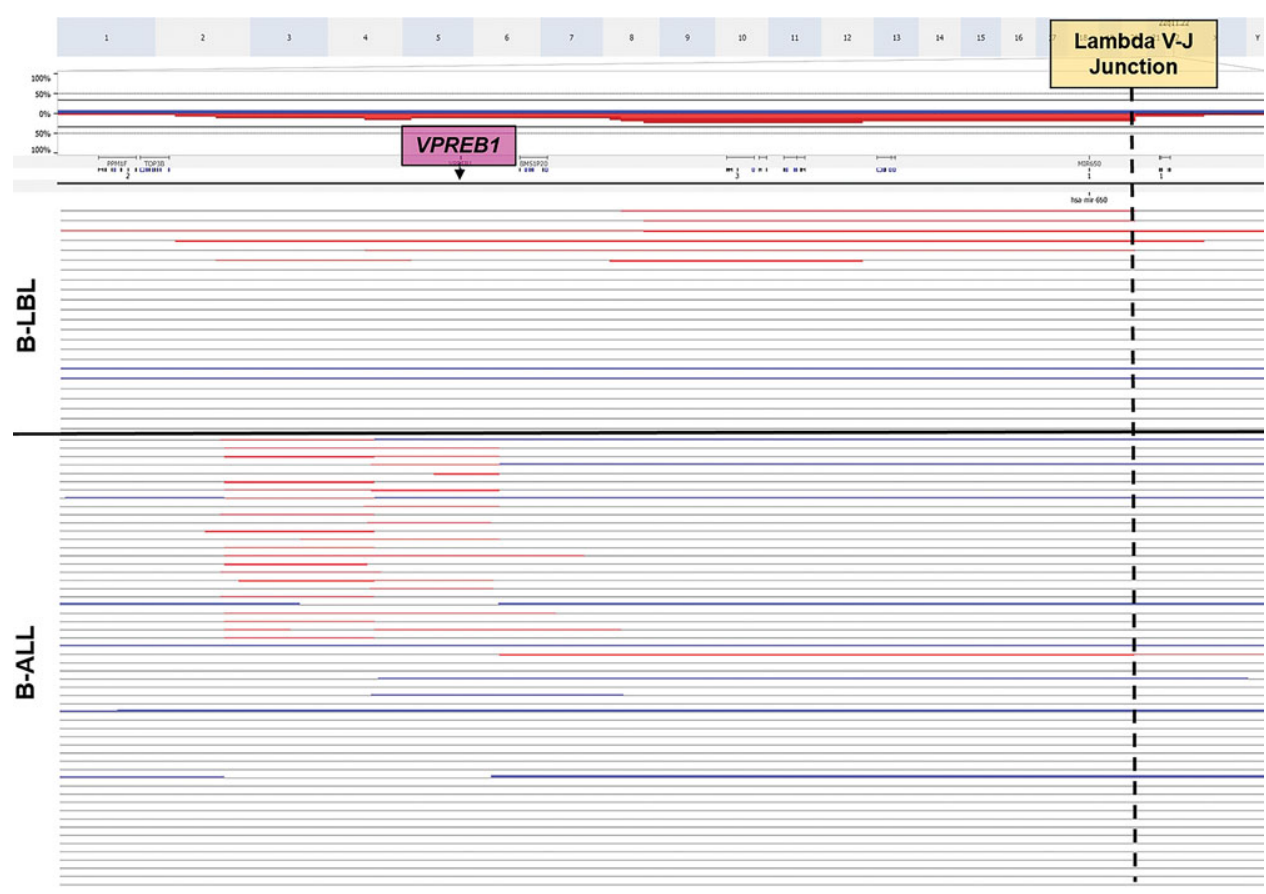

FIGURE 4 B-LBL 22q11.22 deletions resemble the deletion pattern associated with normal immunoglobulin lambda light chain rearrangement. Deletions commonly seen in B-ALL are typically focal and rarely involve the lambda $\mathrm{V}-\mathrm{J}$ function ( $\mathrm{red}=$ deletion; blue = gain)

had massive aneuploidy: one patient showed a near tetraploid karyotype (84 chromosomes) and the other patient was hyperdiploid. ${ }^{14}$ Finally, a study by Schraders et al. ${ }^{15}$ compared B-ALL and B-LBL using snap-frozen specimens from seven cases of B-LBL on Affymetrix SNP array $\mathrm{CGH}$. They detected chromosomal aneuploidies in all seven BLBL cases, and five of the cases (71\%) showed high-hyperdiploidy with 51-54 chromosomes. This rate is higher than that reported by Maitra et al., and although the difference in testing methods might contribute to this discrepancy, the inconsistency is most likely caused by the small sample number in all of the reported studies on B-LBL.

In our current study, by using FFPE tissue sections on archived glass slides from two national clinical trials, we found that B-LBL shares some genomic features with B-ALL including high frequencies of trisomy 21 and deletion of key genes (e.g., CDKN2A, IKZF1, and PAX5). We demonstrated a trend toward less frequent deletion of $B$-cell development genes in B-LBL versus B-ALL, but this may in part be related to the relative higher frequency of hyperdiploidy (43\%), which in BALL is associated with a decreased incidence of such deletions. ${ }^{7}$ Our report of higher rates of hyperdiploidy is in agreement with that of Schraders et al. ${ }^{15}$ and helps to validate hyperdiploidy as a hallmark feature of B-LBL. However, the hyperdiploidy of B-LBL is distinct from that of B-ALL, as triple trisomy $(4,10$, and 17$)$ cases were not identified.

Previous studies of T lineage ALL and LBL identified differences in loss of heterozygosity patterns on chromosome $6 q$ and differences in gene expression profiles between T-LBL and T-ALL. ${ }^{19-21}$ In our study, we have now likewise identified differences between B-LBL and B-ALL. The pattern of deletions within the IGL locus on 22q11.22 in B-LBL is also distinct from what has been seen in B-ALL, where we have previ- ously showed a high incidence of focal IGL deletions that are abnormal and do not represent immunoglobulin light chain rearrangement. Such focal deletions were not seen in B-LBL; however, contiguous IGL deletions, which are consistent with normal Ig light chain rearrangement as seen in normal mature B cells or mature B-cell lymphomas (e.g., Burkitt lymphoma), were identified in B-LBL (22\%) despite being very rare in $B-A L L(2 \%)$. These findings indicate that B-LBL shows genomic features distinct from B-ALL that could indicate pathogenic differences. Although further study will be required, these differences could indicate that B-LBL is derived from a slightly more mature stage of B-cell than B-ALL.

In summary, we performed SNP microarray analysis utilizing FFPE B-ALL and B-LBL samples. Our B-ALL CNA results are similar to those found in fresh-frozen B-ALL tissue cohorts, and our B-LBL findings indicate genomic similarities as well as differences between B-ALL and B-LBL. The significant genomic similarities suggest that treatment of B-LBL according to B-ALL protocols may be appropriate, but this will need to be confirmed in future prospective clinical trials that include genomic characterization of B-LBL. This is the largest high-resolution SNP microarray study of B-LBL to date and the first such study utilizing clinically archived FFPE B-ALL samples. This approach could be of particular value in the future for studying other pediatric lymphomas as well as uncommon B-ALL presentations for which frozen tissues are not available.

\section{ACKNOWLEDGMENTS}

B-LBL patient specimens were provided by the Children's Oncology Group (COG) from the A5971 and AALL0932 clinical trials. We would like to thank Elizabeth Raetz for reading the manuscript and providing 
input. This work was supported in part by a translational research grant from the Leukemia and Lymphoma Society (RRM and JDS).

\section{AUTHOR CONTRIBUTION}

JAM analyzed the data, performed statistical analysis, and wrote the manuscript. JD and ZH analyzed the data. DZ analyzed data and wrote the manuscript. CCM provided statistical and genomic technology advice. VR performed research. AA, MA, BW, PB, MAL, and AT performed research. SLP supported clinical trials, performed research, and wrote manuscript. JDS and RRM designed and directed the research, analyzed the data, and wrote the manuscript.

\section{CONFLICTS OF INTEREST}

The authors declare that there is no conflict of interest.

\section{REFERENCES}

1. Minard-Colin V, Brugieres L, Reiter A, et al. Non-Hodgkin lymphoma in children and adolescents: progress through effective collaboration, current knowledge, and challenges ahead. J Clin Oncol. 2015;33(27):2963-2974.

2. Swerdlow SH, Campo, E, Harris, N.L., et al. WHO Classification of Tumours of Haematopoietic and Lymphoid Tissues. 4th ed. Lyon, France: International Agency for Research on Cancer (IARC); 2008.

3. Maitra A, McKenna RW, Weinberg AG, et al. Precursor B-cell lymphoblastic lymphoma. A study of nine cases lacking blood and bone marrow involvement and review of the literature. Am J Clin Pathol. 2001;115(6):868-875.

4. Lin P, Jones D, Dorfman DM, et al. Precursor B-cell lymphoblastic lymphoma: a predominantly extranodal tumor with low propensity for leukemic involvement. Am J Surg Pathol. 2000;24(11):1480-1490.

5. Mullighan CG, Collins-Underwood JR, Phillips LA, et al. Rearrangement of CRLF2 in B-progenitor- and down syndrome-associated acute lymphoblastic leukemia. Nat Genet. 2009;41(11):1243-1246.

6. Mullighan CG, Miller CB, Radtke I, et al. BCR-ABL1 lymphoblastic leukaemia is characterized by the deletion of Ikaros. Nature. 2008;453(7191):110-114.

7. Mullighan CG, Goorha S, Radtke I, et al. Genome-wide analysis of genetic alterations in acute lymphoblastic leukaemia. Nature. 2007;446(7137):758-764.

8. Mullighan CG, Su X, Zhang J, et al. Deletion of IKZF1 and prognosis in acute lymphoblastic leukemia. N Engl J Med. 2009;360(5):470-480.

9. Mangum DS, Downie J, Mason CC, et al. VPREB1 deletions occur independent of lambda light chain rearrangement in childhood acute lymphoblastic leukemia. Leukemia. 2014;28(1):216-220.

10. Schiffman JD, Lorimer PD, Rodic V, et al. Genome wide copy number analysis of paediatric Burkitt lymphoma using formalin-fixed tissues reveals a subset with gain of chromosome 13q and corresponding miRNA over expression. Br J Haematol. 2011;155(4):477-486.

11. Termuhlen AM, Smith LM, Perkins SL, et al. Disseminated lymphoblastic lymphoma in children and adolescents: results of the COG A5971 trial: a report from the Children's Oncology Group. Br J Haematol. 2013;162(6):792-801.
12. Paulsson K, Forestier E, Lilljebjorn $\mathrm{H}$, et al. Genetic landscape of high hyperdiploid childhood acute lymphoblastic leukemia. Proc Natl Acad SciU SA. 2010;107(50):21719-21724.

13. Sutcliffe MJ, Shuster JJ, Sather HN, et al. High concordance from independent studies by the Children's Cancer Group (CCG) and Pediatric Oncology Group (POG) associating favorable prognosis with combined trisomies 4, 10, and 17 in children with $\mathrm{NCI}$ Standard-Risk B-precursor Acute Lymphoblastic Leukemia: a Children's Oncology Group (COG) initiative. Leukemia. 2005;19(5):734-740.

14. Lones MA, Heerema NA, Le Beau MM, et al. Chromosome abnormalities in advanced stage lymphoblastic lymphoma of children and adolescents: a report from CCG-E08. Cancer Genet Cytogenet. 2007;172(1):111.

15. Schraders M, van Reijmersdal SV, Kamping EJ, et al. High-resolution genomic profiling of pediatric lymphoblastic lymphomas reveals subtle differences with pediatric acute lymphoblastic leukemias in the Blineage. Cancer Genet Cytogenet. 2009;191(1):27-33.

16. Kuiper RP, Schoenmakers EF, Reijmersdal van SV, et al. Highresolution genomic profiling of childhood ALL reveals novel recurrent genetic lesions affecting pathways involved in lymphocyte differentiation and cell cycle progression. Leukemia. 2007;21(6):12581266.

17. Schiffman JD, Wang Y, McPherson LA, et al. Molecular inversion probes reveal patterns of 9p21 deletion and copy number aberrations in childhood leukemia. Cancer Genet Cytogenet. 2009;193(1):9-18.

18. Yang JJ, Bhojwani D, Yang W, et al. Genome-wide copy number profiling reveals molecular evolution from diagnosis to relapse in childhood acute lymphoblastic leukemia. Blood. 2008;112(10):4178-4183.

19. Basso K, Mussolin L, Lettieri A, et al. T-cell lymphoblastic lymphoma shows differences and similarities with T-cell acute lymphoblastic leukemia by genomic and gene expression analyses. Genes Chromosomes Cancer. 2011;50(12):1063-1075.

20. Burkhardt B, Moericke A, Klapper W, et al. Pediatric precursor T lymphoblastic leukemia and lymphoblastic lymphoma: differences in the common regions with loss of heterozygosity at chromosome $6 \mathrm{q}$ and their prognostic impact. Leuk Lymphoma. 2008;49(3):451-461.

21. Raetz EA, Perkins SL, Bhojwani D, et al. Gene expression profiling reveals intrinsic differences between $\mathrm{T}$-cell acute lymphoblastic leukemia and T-cell lymphoblastic lymphoma. Pediatr Blood Cancer. 2006;47(2):130-140.

\section{SUPPORTING INFORMATION}

Additional Supporting Information may be found online in the supporting information tab for this article.

How to cite this article: Meyer JA, Zhou D, Mason CC, Downie JM, Rodic V, Abromowitch M, Wistinghausen B, Termuhlen AM, Angiolillo AL, Perkins SL, Barnette P, Schiffman JD, Miles RR. Genomic characterization of pediatric B-lymphoblastic lymphoma and B-lymphoblastic leukemia using formalin-fixed tissues. Pediatr Blood Cancer. 2017;64: e26363. doi: 10.1002/pbc.26363 